\title{
Edge Detection: A Statistical approach
}

\author{
Amiya Halder, Nilabha Chatterjee, Arindam Kar, Swastik Pal and Soumajit Pramanik \\ Department of Computer Science \& Engineering \\ St. Thomas' College of Engineering \& Technology, \\ Kolkata-23, West Bengal, India \\ amiya_halder@indiatimes.com,mr.nilabha@gmail.com,soumajit.pramanik@gmail.com
}

\begin{abstract}
This paper describes a novel edge detection algorithm for gray scale images. The proposed method is based on the neighborhood similarity of a pixel using a pre-defined intensity range and simple statistical approach. Then using three or four neighboring boundary pixel to detect a noise and reduced this noise. Many experiments were carried out to evaluate and compare the performance of the proposed algorithm. This new detector outperforms the previously available classical edge detectors.
\end{abstract}

Keywords- Edge Detection; Sobel Operator; Prewitt Operator; Roberts Operator; Noise Reduction.

\section{INTRODUCTION}

Edges are boundaries between different textures. Edge also can be defined as discontinuities in image intensity from one pixel to another. The edges for an image are always the important characteristics that offer an indication for a higher frequency. Detection of edges for an image may help for image segmentation, data compression, and also help for well matching, such as image reconstruction and so on $[1,2]$. Variables involved in the selection of an edge detection operator include Edge orientation, Noise environment and Edge structure. The geometry of the operator determines a characteristic direction in which it is most sensitive to edges. Operators can be optimized to look for horizontal, vertical, or diagonal edges. Edge detection is difficult in noisy images, since both the noise and the edges contain high-frequency content. Attempts to reduce the noise result in blurred and distorted edges. Operators used on noisy images are typically larger in scope, so they can average enough data to discount localized noisy pixels. This results in less accurate localization of the detected edges. Not all edges involve a step change in intensity. Effects such as refraction or poor focus can result in objects with boundaries defined by a gradual change in intensity. The operator needs to be chosen to be responsive to such a gradual change in those cases. So, there are problems of false edge detection, missing true edges, edge localization, high computational time and problems due to noise etc. Therefore, the objective is to do the comparison of various edge detection techniques and analyze the performance of the various techniques in different conditions. In the field of image segmentation, there are many methods for edge detection based on different algorithms namely, derivative methods, surface methods, morphological methods, adaptive methods and structural methods.

In this paper we present a new approach to detect edges of a gray scale images. This approach consists of two steps. At step1, determine the all edge pixels for edge detection of the image and in step2, remove impulsive noise from the output of step 1 to enhance the edge map.

The rest of this paper is organized as follows: - in section II, the some classical edge detection technique is provided. Section III gives the concepts of the proposed algorithm and section IV described the experimental results and section V concludes the paper.

\section{IMAGE EDGE DETECTION}

Edge detection refers to the extraction of the edges in a digital image. It is a process whose aim is to identify the points in an image where discontinuities or sharp changes in intensity occur. This process is crucial to understanding the content of an image and has its applications in image analysis and machine vision. It is usually applied in initial stages of computer vision applications. Edge detection aims to localize the boundaries of objects in an image and is a basis for many image analysis and machine vision applications. Conventional approaches (such as Sobel Operator, Prewitt Operator, Roberts Operator etc.) to edge detection are computationally expensive because each set of operations is conducted for each pixel $[1,2]$. In conventional approaches, the computation time quickly increases with the size of the image. A statistical-based approach has the potential of overcoming the limitations of conventional methods. Furthermore, it makes the algorithm easily adaptable for any systems. Various edge detection techniques have been developed for edge detection [4-9].

\section{PROPOSED ALGORITHM}

We have proposed an efficient edge detection algorithm using simple mathematical approach. This proposed method depends on masking operation. A $5 \times 5$ mask centered at pixel $\mathrm{f}(\mathrm{i}, \mathrm{j})$ is defined as shown in given below:

\begin{tabular}{|c|c|c|c|c|}
\hline$f(i-2, j-2)$ & $f(i-2, j-1)$ & $f(i-2, j)$ & $f(i-2, j+1)$ & $f(i-2, j+2)$ \\
\hline$f(i-1, j-2)$ & $f(i-1, j-1)$ & $f(i-1, j)$ & $f(i-1, j+1)$ & $f(i-1, j+2)$ \\
\hline$f(i, j-2)$ & $f(i, j-1)$ & $\mathbf{f}(\mathbf{i}, j)$ & $f(i, j+1)$ & $f(i, j+2)$ \\
\hline$f(i+1, j-2)$ & $f(i+1, j-1)$ & $f(i+1, j)$ & $f(i+1, j+1)$ & $f(i+1, j+2)$ \\
\hline$f(i+2, j-2)$ & $f(i+2, j-1)$ & $f(i+2, j)$ & $f(i+2, j+1)$ & $f(i+2, j+2)$ \\
\hline
\end{tabular}




\section{A. Edge Detection}

In this proposed method, using neighboring boundary pixel determines the all edge pixels for edge detection of the image. We considered only 8 bit gray scale image in any arbitrary dimension.

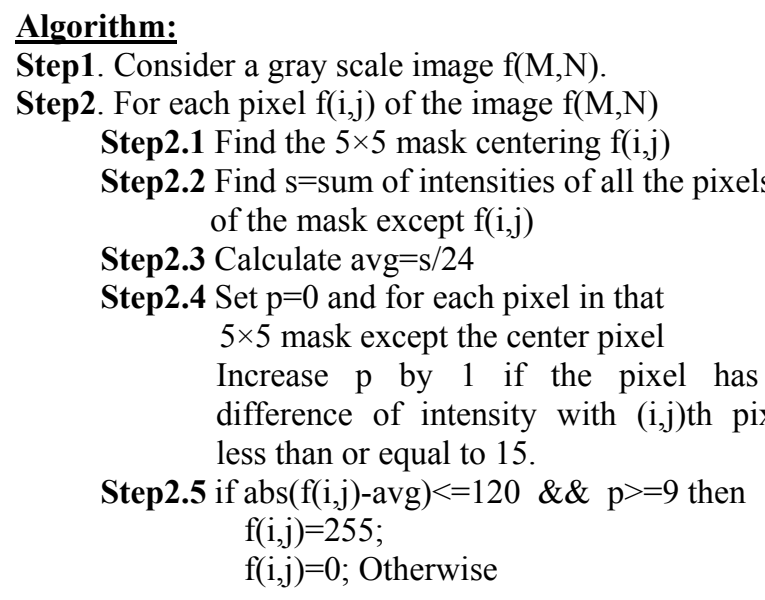

\section{B. Noise Detection}

Noise reduction is one of the most important aspects in order to improve image quality. There exist different types of noises (Speckle noise, Gaussian noise, Impulsive noise etc), which damage image quality. This technique is limited only on impulsive noise (or Salt and pepper noise) reduction from binary images (or edge map of a gray scale image). In this paper our approach is greedy one, which is we consider a spoiled pixel (noise) and replace it with the pixel value of its surrounding 4 or 3 neighbors.

Suppose there is a white point in a black background. So, check for its neighbor's pixel value, if all of its 4 neighbor's pixel value is 0 then we also place a 0 on the white point (255). This approach is simply the vice-versa in case of a black pixel in a white background.

If the input image contains much more noise, then both 4 and 3 neighboring pixel values can be considered.

\section{Algorithm:}

Step1. Take a binary image $\mathrm{f}(\mathrm{M}, \mathrm{N})$

Step2. For all pixel $p \in f(M, N)$ do

Step2.1 If a pixel pi(f(i,j)) $\in \mathrm{f}(\mathrm{M}, \mathrm{N})$ is black, then check its neighbor pixel pj, where pj $\in\{f(i, j-1), f(i, j+1), f(i-1, j), f(i+1, j)\}$

Step2.1.1 If all 4 (or 3) neighbors are white, then make the considered point pi is white;

Step2.1.2 Otherwise do nothing;

Step2.2 If the pixel pi(f(i,j)) is white, then check its neighbor pixel pj, where pj $\in\{f(i, j-1), f(i, j+1), f(i-1, j), f(i+1, j)\}$

Step2.2.1 If all 4 (or 3) neighbors are black, then make the considered point pi is black;

Step2.2.2 Otherwise do nothing;
This algorithm performs on some of the standard images. We considered both black and white points and also 4 and 3 neighboring pixels for checking.

\section{EXPERIMENTAL RESULTS}

This algorithm developed has been simulated using MATLAB. The input images are considered to be .pgm images. The precision is assumed to be 8 i.e. the number of bits per pixel is 8 . All the image files that we have tested are of different sizes.

Various evaluation techniques of edge map have been developed $[3,11]$. Comparison of an edge map, obtained by a detector of edges [11], with its ground truth can be achieved through a set of direct measurements, such as the number of correctly detected edge pixels, called true positive (TP), the number of pixels erroneously classified as edge pixels, called false positive (FP), the amount of edge pixels that were not classified as edge pixel, called false negative (FN). From these measures, the following statistical indices have been proposed:

The percentage of pixels that were correctly detected $\left(\mathrm{P}_{\mathrm{CO}}\right)$ :

$$
P_{c o}=\frac{T P}{\max \left(N_{I}, N_{B}\right)}--------(1)
$$

Where $\mathrm{N}_{I}$ represents the number of edge points of the ideal image and $\mathrm{N}_{\mathrm{B}}$ the number of edge points detected. The percentage of pixels that were not detected $\left(\mathrm{P}_{\mathrm{nd}}\right)$ :

$$
P_{n d}=\frac{F N}{\max \left(N_{I}, N_{B}\right)}--------(2)
$$

The percentage of pixels that were erroneously detected as edge pixels, i.e. the percentage of false alarm $\left(\mathrm{P}_{\mathrm{fa}}\right)$ :

$$
P_{f a}=\frac{F P}{\max \left(N_{I}, N_{B}\right)}--------(3)
$$

The figure of merit of Pratt is another useful measure for assessing the performance of edge detectors. This measure uses the distance between all pairs of points corresponding to quantify, with precision, the difference between the contours [10]. The figure of merit of Pratt, which assesses the similarity between two contours, is defined as:

$$
I M P=\frac{1}{\max \left(N_{I}, N_{B}\right)} \sum_{1}^{N_{B}} \frac{1}{1+\alpha \times d_{i}^{2}}---(4),
$$

Where $\mathrm{N}_{\mathrm{I}}$ and $\mathrm{N}_{\mathrm{B}}$ are the points of edges in the image and ground truth image, respectively, $d_{i}$ is the distance between a edge pixel and the nearest edge pixel of the ground truth and $\alpha$ is a empirical calibration constant and was used $\alpha=$ $1 / 9$, optimal value established by Pratt [10]. The figure of merit of Pratt IMP is an indicator of the quality of edge, and 
reflects the overall behavior of the distances between the edges, being a relative measure, which varies in the range $[0,1]$, where 1 represents the optimal value, i.e., the edges detected coincide with the ground truth.

The values of statistical indices represented by the equations (1), (2) and (3) ranging between 0 and 1, and reach ideal values in case 1 for $\mathrm{P}_{\mathrm{CO}}$ and 0 for indices $\mathrm{P}_{\text {nd }}$ and $\mathrm{P}_{\mathrm{fa}}$. From the combination of the indices defined by equations (1), (2) and (3), together with the index of merit of Pratt (equation 4), has proposed a new global index, which is defined by Euclidean distance $\left(d_{f 2}^{4}\right)$ in $\mathrm{R}^{4}$ to the point $\mathrm{P}=(1 ; 1 ; 0 ; 0)$, where its coordinates are optimum values achieved by indices $\mathrm{P}_{\mathrm{CO}}$, IMP, $\mathrm{P}_{\mathrm{nd}}$ and $\mathrm{P}_{\mathrm{fa}}$ respectively. The point $\mathrm{P}$ represents the optimum point to be reached by an ideal edge detector. The distance to this point can be calculated by the equation (5):

$d_{\jmath_{2}}^{4}=\sqrt{(}\left(P_{c o}-1\right)^{2}+(I M P-1)^{2}+P_{n d}^{2}+P_{f a}^{2}---(5)$

The distance defined $d_{f 2}^{4}$ varies between 0 and 2 , where the value 0 represents the perfect fit for this measure, i.e., the best edge detector among several detectors will minimize this distance.

This proposed method gives the better result comparison with Roberts, Prewitt and Sobel operator are shown in TABLE I -TABLE VIII for different images.

The proposed algorithm has been applied to well known natural images such as Lena, MRI and peppers etc. Figure1 are shown the some output images using this proposed edge detection method.

TABLE I. QUANTITATIVE MEASURES OBTAINED BY EDGE DETECTORS FOR LENA IMAGE

\begin{tabular}{|c|c|c|c|c|}
\hline & Proposed & Sobel & Roberts & Prewitt \\
\hline IMP & 0.8484 & 0.3406 & 0.3271 & 0.3383 \\
\hline FP & 30935 & 1784 & 3172 & 1722 \\
\hline FN & 8870 & 13186 & 14662 & 13171 \\
\hline $\mathrm{TP}$ & 8715 & 4399 & 2923 & 4414 \\
\hline $\mathrm{P}_{\text {nd }}$ & 0.7802 & 0.1015 & 0.1804 & 0.0979 \\
\hline $\mathrm{P}_{\mathrm{co}}$ & 0.2198 & 0.2502 & 0.1662 & 0.2510 \\
\hline $\mathrm{P}_{\mathrm{fa}}$ & 0.2237 & 0.7498 & 0.8338 & 0.7490 \\
\hline $\mathrm{d}_{\mathrm{f} 2}^{4}$ & 1.1360 & 1.2529 & 1.3696 & 1.2528 \\
\hline
\end{tabular}

TABLE II. QUANTITATIVE MEASURES OBTAINED BY EDGE DETECTORS FOR WEEL IMAGE

\begin{tabular}{|c|c|c|c|c|}
\hline & Proposed & Roberts & Prewitt & Sobel \\
\hline IMP & 0.7945 & 0.2889 & 0.2325 & 0.2329 \\
\hline $\mathrm{FP}$ & 28570 & 1870 & 799 & 791 \\
\hline $\mathrm{FN}$ & 9366 & 13284 & 13248 & 13234 \\
\hline $\mathrm{TP}$ & 6958 & 3040 & 3076 & 3090 \\
\hline $\mathrm{P}_{\text {nd }}$ & 0.8042 & 0.1146 & 0.0489 & 0.0485 \\
\hline $\mathrm{P}_{\mathrm{co}}$ & 0.1958 & 0.1862 & 0.1884 & 0.1893 \\
\hline $\mathrm{P}_{\mathrm{fa}}$ & 0.2636 & 0.8138 & 0.8116 & 0.8107 \\
\hline $\mathrm{d}_{\mathrm{f} 2}^{4}$ & 1.1854 & 1.3576 & 1.3816 & 1.3803 \\
\hline
\end{tabular}

TABLE III. QUANTITATIVE MEASURES OBTAINED BY EDGE DETECTORS FOR PEPPERS IMAGE

\begin{tabular}{|c|c|c|c|c|}
\hline & Proposed & Roberts & Prewitt & Sobel \\
\hline $\mathrm{IMP}$ & 0.8176 & 0.3328 & 0.3297 & 0.3307 \\
\hline $\mathrm{FP}$ & 32780 & 3585 & 2005 & 2076 \\
\hline $\mathrm{FN}$ & 9520 & 15192 & 13848 & 13891 \\
\hline $\mathrm{TP}$ & 8530 & 2858 & 4202 & 4159 \\
\hline $\mathrm{P}_{\mathrm{nd}}$ & 0.7935 & 0.1986 & 0.1111 & 0.1150 \\
\hline $\mathrm{P}_{\mathrm{co}}$ & 0.2065 & 0.1583 & 0.2328 & 0.2304 \\
\hline $\mathrm{P}_{\mathrm{fa}}$ & 0.2305 & 0.8417 & 0.7672 & 0.7696 \\
\hline $\mathrm{d}_{\mathrm{f} 2}^{4}$ & 1.1600 & 1.3789 & 1.2802 & 1.2829 \\
\hline
\end{tabular}

TABLE IV. QUANTITATIVE MEASURES OBTAINED BY EDGE DETECTORS FOR PARROT IMAGE

\begin{tabular}{|c|c|c|c|c|}
\hline & Proposed & Roberts & Prewitt & Sobel \\
\hline IMP & 0.8278 & 0.3312 & 0.4023 & 0.4073 \\
\hline $\mathrm{FP}$ & 32904 & 3970 & 3040 & 3215 \\
\hline $\mathrm{FN}$ & 9361 & 15616 & 13477 & 13533 \\
\hline $\mathrm{TP}$ & 8813 & 2558 & 4697 & 4641 \\
\hline $\mathrm{P}_{\text {nd }}$ & 0.7887 & 0.2184 & 0.1673 & 0.1769 \\
\hline $\mathrm{P}_{\mathrm{co}}$ & 0.2113 & 0.1408 & 0.2584 & 0.2554 \\
\hline $\mathrm{P}_{\mathrm{fa}}$ & 0.2244 & 0.8592 & 0.7416 & 0.7446 \\
\hline $\mathrm{d}_{\mathrm{f} 2}^{4}$ & 1.1508 & 1.4041 & 1.2186 & 1.2213 \\
\hline
\end{tabular}

TABLE V. QUANTITATIVE MEASURES OBTAINED BY EDGE DETECTORS FOR ZELDA IMAGE

\begin{tabular}{|c|c|c|c|c|}
\hline & Proposed & Roberts & Prewitt & Sobel \\
\hline IMP & 0.8212 & 0.2752 & 0.3494 & 0.3499 \\
\hline $\mathrm{FP}$ & 24563 & 3681 & 3353 & 3366 \\
\hline $\mathrm{FN}$ & 14919 & 18750 & 16872 & 16872 \\
\hline $\mathrm{TP}$ & 6573 & 2742 & 4620 & 4620 \\
\hline $\mathrm{P}_{\text {nd }}$ & 0.7889 & 0.1713 & 0.1560 & 0.1566 \\
\hline $\mathrm{P}_{\mathrm{co}}$ & 0.2111 & 0.1276 & 0.2150 & 0.2150 \\
\hline $\mathrm{P}_{\mathrm{fa}}$ & 0.4792 & 0.8724 & 0.7850 & 0.7850 \\
\hline $\mathrm{d}_{\mathrm{f} 2}^{4}$ & 1.2273 & 1.4411 & 1.2962 & 1.2960 \\
\hline
\end{tabular}

TABLE VI. QUANTITATIVE MEASURES OBTAINED BY EDGE DETECTORS FOR TOOL IMAGE

\begin{tabular}{|c|c|c|c|c|}
\hline & Proposed & Roberts & Prewitt & Sobel \\
\hline IMP & 0.7755 & 0.2087 & 0.2185 & 0.2183 \\
\hline FP & 23848 & 2385 & 935 & 940 \\
\hline FN & 15176 & 19535 & 18020 & 18028 \\
\hline TP & 6806 & 2447 & 3962 & 3954 \\
\hline $\mathrm{P}_{\mathrm{nd}}$ & 0.7780 & 0.1085 & 0.0425 & 0.0428 \\
\hline $\mathrm{P}_{\mathrm{c}}$ & 0.2220 & 0.1113 & 0.1802 & 0.1799 \\
\hline $\mathrm{P}_{\mathrm{fa}}$ & 0.4951 & 0.8887 & 0.8198 & 0.8201 \\
\hline $\mathrm{d}_{\mathrm{f} 2}^{4}$ & 1.2272 & 1.4891 & 1.3988 & 1.3993 \\
\hline
\end{tabular}

TABLE VII. QUANTITATIVE MEASURES OBTAINED BY EDGE DETECTORS FOR MRI IMAGE

\begin{tabular}{|c|c|c|c|c|}
\hline & Proposed & Roberts & Prewitt & Sobel \\
\hline IMP & 0.7899 & 0.2231 & 0.1937 & 0.1922 \\
\hline FP & 20978 & 1871 & 804 & 805 \\
\hline FN & 10025 & 13448 & 12939 & 12962 \\
\hline TP & 5132 & 1709 & 2218 & 2195 \\
\hline$P_{\text {nd }}$ & 0.8034 & 0.1234 & 0.0530 & 0.0531 \\
\hline $\mathrm{P}_{\mathrm{co}}$ & 0.1966 & 0.1128 & 0.1463 & 0.1448 \\
\hline $\mathrm{P}_{\mathrm{fa}}$ & 0.3840 & 0.8872 & 0.8537 & 0.8552 \\
\hline $\mathrm{d}_{\mathrm{f} 2}^{4}$ & 1.2176 & 1.4809 & 1.4527 & 1.4553 \\
\hline
\end{tabular}




\section{CONCLUSIONS}

A novel approach to image processing utilizing edge detection and reduced the noise within images has been introduced within this paper. This approach gives the better results than the classical edge detection techniques. In future this technique will be applied in colour images. The hypothesis, on which, this work was based, was shown to be mathematically feasible.

\section{REFERENCES}

[1] Pratt, William K. " Digital Image Processing”.

[2] Rafael C. Gonzalez, Richard E. Woods, Digital Image Processing, Pearson Education, 2002

[3] Mamta Juneja , Parvinder Singh Sandhu, Performance Evaluation of Edge Detection Techniques for Images in Spatial Domain, International Journal of Computer Theory and Engineering, Vol. 1, No. 5, December, 2009.

[4] M. H. Hueckel. "A local visual operator which recognizes edges and line”. J. ACM, vol. 20, no. 4, pp. 634-647, Oct. 1973.

[5] Y. Yakimovsky, "Boundary and object detection in real world images".JACM, vol. 23, no. 4, pp. 598-619, Oct. 1976.
[6] D. Marr and E.Hildreth. "Theory of Edge Detection". Proceedings of the Royal Society of London. Series B, Biological Sciences,, Vol. 207, No.1167. (29 February 1980), pp. 187-217.

[7] M. Heath, S. Sarkar, T. Sanocki, and K.W. Bowyer. "A Robust Visual Method for Assessing the Relative Performance of Edge Detection Algorithms". IEEE Trans. Pattern Analysis and Machine Intelligence, vol. 19, no. 12, pp. 1338-1359, Dec. 1997.

[8] Noor Elaiza Abdul Khalid, Mazani Manaf, Mohd Ezane Aziz, “An Empirical Study using Line Profile Histogram Approximation of Edge Detection Algorithms", ICCEA 2010,pp.344-348.

[9] N. Senthilkumaran and R. Rajesh, "Edge Detection Techniques for Image Segmentation - A Survey of Soft Computing Approaches", International Journal of Recent Trends in Engineering, Vol. 1, No. 2, May 2009.

[10] I. A. Abdou and W. Pratt, "Quantitative design and evaluation of enhancement/thresholding edge detectors," in Proceedings of the IEEE, vol. 67, no. 5, 1979, pp. 753-766.

[11] Ines Aparecida Gasparotto Boaventura, Adilson Gonzaga, “ Method to Evaluate the Performance of Edge Detector", International Conference on Intelligent Systems Design and Applications, pp. 341346, 2007.

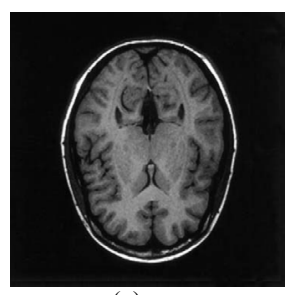

(a)

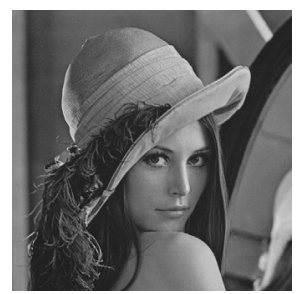

(f)

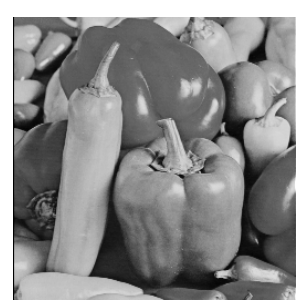

(k)

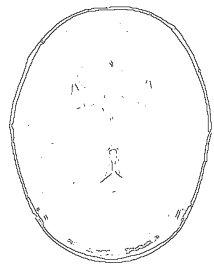

(b)

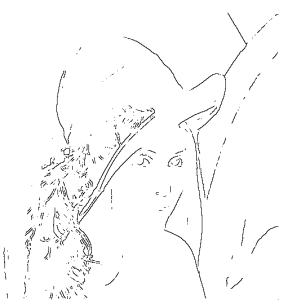

(g)

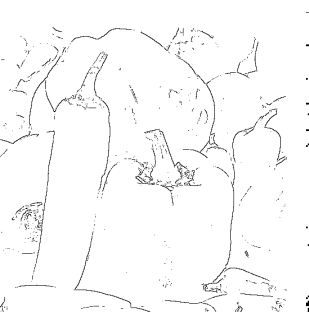

(1)

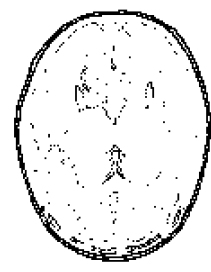

(c)

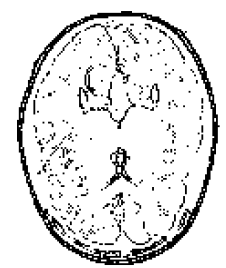

(d)

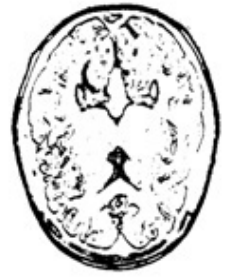

(e)

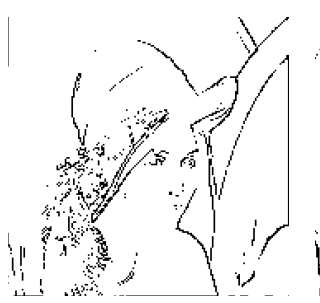

(h)

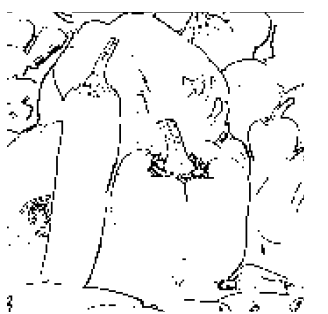

(m)

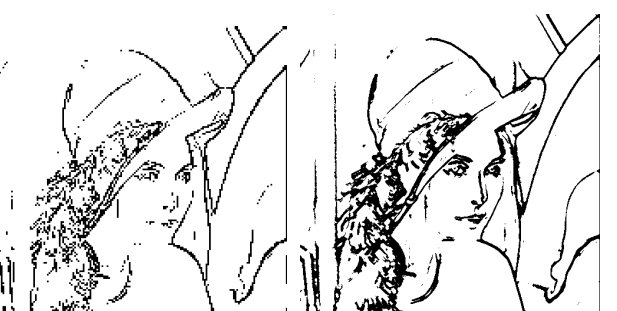

(i)

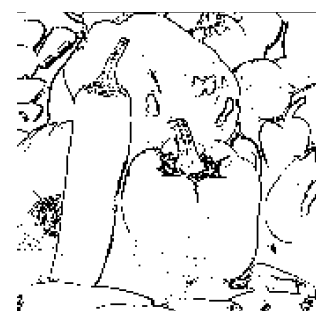

(n) (j)

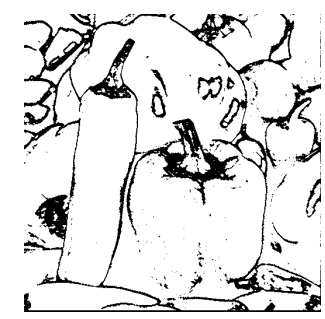

(o)

Figure 1: Experimental results for some well known images: (a),(f),(k)-Original Image, (b),(g),(l)- Edge detection using Robert operator, (c),(h),(m)- Edge detection using Prewitt operator, (d),(i),(n)- Edge detection using Sobel operator,(e),(j),(o)- Edge detection using Proposed method. 